\title{
Supplementary Material on
}

\section{Concepts on the Protein Folding Problem}

\author{
Jean-Numa Gillet $^{1}$ and Indira Ghosh ${ }^{2}$ \\ Journal of Biomolecular Structure and Dynamics, Manuscript Number: [Insert Number]
}

\section{1) Additional explanations on the hydrophobic, hydrophilic and flexibility effects}

The three effects of the forces related to hydrophobicity, hydrophilicity and flexibility exerted on a protein are necessary for its folding (especially for non-small proteins). First, hydrophobicity enables compactness of the 3D structure of the protein by burying hydrophobic backbone parts but also side-chain groups to its core. Thus, the number of really important reaction coordinates to describe the $3 \mathrm{D}$ structure of the protein decreases during folding to its native state and make the protein stiffer, increasing stability of its core. At the same time, the external surface of the protein minimizes to reduce the number of energetically unfavorable hydrophobic groups in contact with water but leaving favorable charged and hydrophilic groups at its surface, which is another mechanism to increase the core stability. Flexibility is however a third important factor leading to the protein's native state. This entropic effect enables to avoid long relaxation times of the protein in a 'frozen' intermediate state $I$. Indeed, state $I$ presents a remaining positive entropy balance $\Delta S$ if the flexibility of its related conformation $\mathbf{C}_{I}$ is large enough. Due to $\Delta S>0$, this state is not so frozen than in current molecular dynamics (MD) models avoiding entropy calculation. Indeed, remaining flexible parts of $\mathbf{C}_{I}$ (as backbone tails and side-chains) can induce a faster kinetics for convergence to $\mathbf{C}_{N}$ of the folding process. Experiments showed that non-native nuclei (as $\alpha$-helix and $\beta$-sheets) can be rapidly dismantled to form other nuclei belonging to the native conformation. Also, mention that kinetic energy (KE) fluctuations are underestimated in MD models because the average $K E$ is computed by assuming each protein's heavy atom as not bound to the others. A more realistic $K E$ calculation would also induce a faster kinetics to escape intermediate states.

\footnotetext{
${ }^{1}$ Email: jngillet@gmail.com

2 Emails: indirag@mail.jnu.ac.in, ighdna@yahoo.com
} 


\section{2) Average side-chain flexibility, hydrophobicity and hydrophilicity characteristics}

The utilized Protein Data Bank (PDB) contains 83,106 experimentally-determined structures. Redundancy was removed with a similarity cutoff of $90 \%$. This means that among clusters of proteins, which share a sequence similarity of $90 \%$, only one representative sequence was incorporated in the dataset to be analyzed. This reduced our dataset to 33,459 folded protein structures. A protein structure can have multiple chains. This dataset contains a total of 75,221 protein chains. The proteins of this dataset have a minimum (backbone) length of 12 residues and maximum length of 930 residues. We computed the side-chain flexibilities for each protein contained in our dataset. The flexibility value of a side-chain simply refers to the number of heavy atoms in the side-chain that are linked to a $\alpha$-Carbon. For serine, two heavy atoms $(\beta$-Carbon and $\gamma$-Oxygen) are linked to the $\alpha$-Carbon. The flexibility of serine is thus of 2. The Kyte-Doolittle scale is used to characterize the degrees of hydrophobicity and hydrophilicity of an amino acid. This scale ranges from -4.5 to +4.5 . Positive or negative values are respectively related to hydrophobic or hydrophilic amino acids. For all proteins in our dataset, we first calculated their lengths (i.e. their amino-acid numbers). Then, the average side-chain flexibility parameter of each protein is computed by adding the flexibility values of all side-chains of the considered protein and normalizing the sum by its respective length. In a similar way, the average hydrophobicity and hydrophilicity values were estimated for each protein. Fig. S1 displays the average side-chain flexibility (red), hydrophobicity (green) and hydrophilicity (black) characteristics with standard deviation bars. Each index (from 1 to 30) of the abscissa axis corresponds to an averaging bin for the proteins in a length range that does not exceed 30 residues of difference. For instance, the proteins cluster in bin No. 1, No. 2, No. 3 or No. 30 have increasing lengths in ranges from 12 to 42, 42 to 72, 72 to 102 or 882 to 912 residues, respectively. As sketched in Fig. S1, we observed that the three characteristics have almost constant values when the bin index is increased from 3 to 30. Therefore, when the protein length is higher than $\sim 70$ to 100 residues, it is not possible to infer a superiority of one of the three effects (hydrophobicity, hydrophilicity or flexibility) over the others at the entire protein scale, when the protein is fully unfolded. 


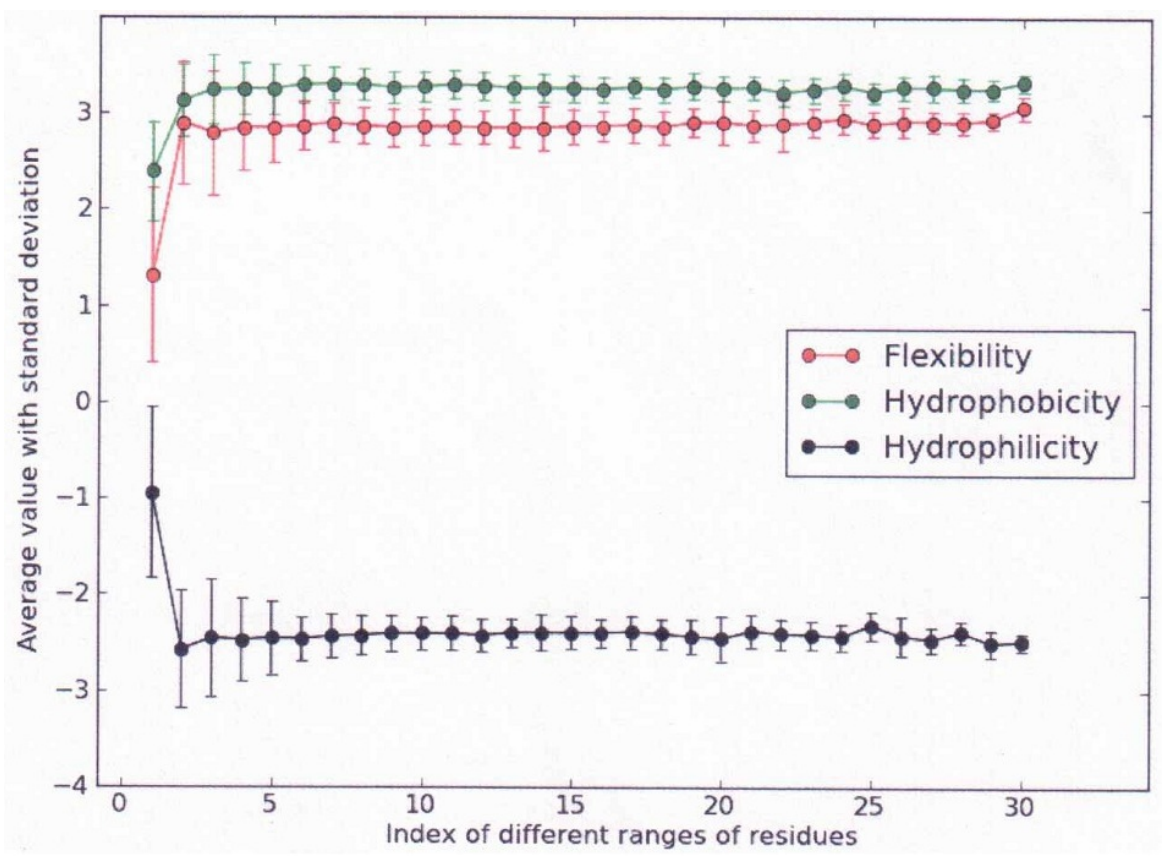

Fig. S1. Average side-chain flexibility (red), hydrophobicity (green) and hydrophilicity (black) characteristics with standard deviation bars. The proteins in bin No. 1, No. 2, No. 3 or No. 30 have increasing lengths in ranges from 12 to 42,42 to 72,72 to 102 or 882 to 912 residues, respectively.

\section{3) Degree of anti-correlation between $\log \left(k_{f}\right)$ of various proteins vs. their lengths}

The logarithms of the experimentally-determined folding rates $k_{f}$ of miscellaneous proteins were collected from the KineticDB database (Bogatyreva et al., 2009) and paper by Ivankov et al. (2009). These experimentalists reported the folding kinetics in water of 88 protein types with lengths varying in a range from 16 to 390 residues. Fig. S2 presents a plot of $\log \left(k_{f}\right)$ vs. the protein length $L$. Each circle corresponds to one experimental measure $\left[L, \log \left(k_{f}\right)\right]$ for one protein taken in the database. The best linear fit is shown by the dashed line. It seems that there is a light anticorrelation between $L$ and $\log \left(k_{f}\right)$. However, the regression coefficient is of only -0.021 and coefficient of determination $\left(R^{2}\right)$ of only 0.40 , quite far from unity for a straight line. Therefore, no clear decrease relationship of $\log \left(k_{f}\right)$ vs. $L$ can be claimed from this correlation analysis. The common previous assumption that $k_{f}$ is reduced when $L$ is increased will not hold for many large proteins. 


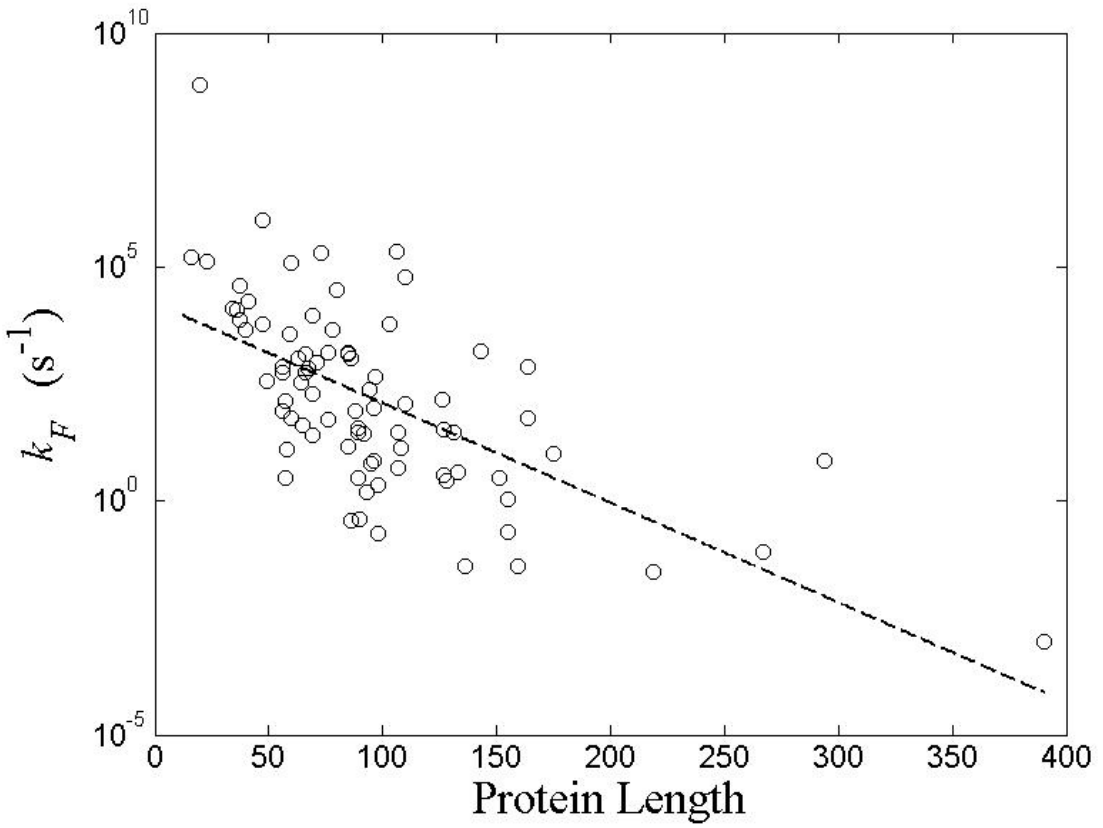

Fig. S2. $\log \left(k_{f}\right)$ vs. $L$ (circles) and linear fit (dashed line).

Acknowledgements: We thank Mr. Tausif. Khan for help in achievement of the data analyses presented in S1 \& S2. 